\title{
Eficiência de inseticidas em tratamento de sementes de milho no controle da cigarrinha Dalbulus maidis (Hemiptera: Cicadellidae) em viveiro telado
}

\author{
Insecticides efficiency in treatment of corn seeds to control leafhopper Dalbulus maidis (Hemiptera: \\ Cicadellidae) in greenhouse
}

Charles Martins de Oliveira ${ }^{\text {I }}$ Elizabeth de Oliveira ${ }^{\text {II }}$ Marcus Canuto ${ }^{\text {III }}$ Ivan Cruz

\begin{abstract}
O objetivo deste trabalho foi verificar a eficiencia de inseticidas no tratamento de sementes no controle da cigarrinha-do-milho em viveiro telado. Sementes de milho foram tratadas ou não com inseticidas (imidacloprid, thiamethoxan, thiodicarb + zn, thiodicarb, carbofuran, carbofuran + zn e carbosulfan) e semeadas em vasos plásticos. Nas plantas, foram confinadas cigarrinhas sadias e avaliada a eficiência desses inseticidas no controle desse inseto, em diferentes intervalos de tempo e em períodos sucessivos. Os produtos imidacloprid e thiamethoxan foram os mais eficientes no controle da cigarrinha, proporcionando eficiência de controle de adultos de D. maidis igual ou superior a $70 \%$, até o trigésimo dia de avaliação, após 4 a 24h de confinamento das cigarrinhas.
\end{abstract}

Palavras-chave: inseticidas, cigarrinha-do-milho, Spiroplasma kunkelii, fitoplasma, Zea mays.

\section{ABSTRACT}

This research was aimed at checking the efficiency of insecticide seed treatment on corn leafhopper control, at greenhouse. Maize seeds were treated with insecticides (imidacloprid, thiamethoxan, thiodicarb $+z n$, thiodicarb, carbofuran, carbofuran $+z n$ and carbosulfan) and sowed on plastic pots. On those plants healthy leafhoppers were confined, and the efficiency of those insecticides on its control was evaluated, at different intervals of time and in successive periods. The insecticides imidacloprid and thiamethoxan were the most efficient to control the corn leafhoppers, and provided control efficiency of $\boldsymbol{D}$. maidis adults equal or upper $70 \%$ until the thirtieth day of evaluation, after 4 to $24 \mathrm{~h}$ of leafhoppers confining.

\author{
Key words: insecticides, corn leafhopper, Spiroplasma \\ kunkelii, fitoplasma, Zea mays.
}

A cigarrinha-do-milho, Dalbulus maidis (DeLong \& Wolcott) (Hemiptera: Cicadellidae), é o vetor dos molicutes fitoplasma e Spiroplasma kunkelii, agentes causais do enfezamento vermelho e do enfezamento pálido, respectivamente, e do Maize Rayado Fino virus (Marafivirus - MRFV) (LOPES \& OLIVEIRA, 2004). Os danos causados por essas doenças na cultura do milho (Zea mays L.) se refletem em prejuízos severos (OLIVEIRA et al., 1998).

D. maidis apresenta alto potencial biótico e migra a longas distâncias (OLIVEIRA, 2000), colonizando campos de milho recém-germinados. A transmissão dos fitopatógenos ocorre em menos de uma hora (LEGRAND \& POWER, 1994), requerendo medidas que interfiram eficientemente nesse processo. O tratamento inseticida de sementes poderia ser uma alternativa para reduzir a população desse inseto-vetor e minimizar a disseminação dos fitopatógenos.

O objetivo deste estudo foi o de avaliar a eficiência de inseticidas em tratamento de sementes de milho no controle da cigarrinha $\boldsymbol{D}$. maidis sob condições de viveiro telado.

O estudo foi conduzido em vasos, em viveiro telado, utilizando-se oito tratamentos, sendo: 1-

IEmbrapa Cerrados, Rod. BR 020, km 18, CP 08223, 73310-970, Planaltina, DF, Brasil. E-mail: charles@cpac.embrapa.br. Autor para correspondência.

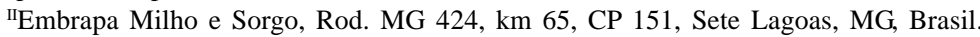

IIInicentro Isabella Hendrix, Belo Horizonte, MG, Brasil. 
testemunha (sem inseticida); 2- thiamethoxan ; 3imidacloprid; 4- thiodicarb + Zn; 5- thiodicarb; 6carbofuran; 7- carbofuran $+\mathrm{Zn}$; 8- carbosulfan nas doses de $0,28 \mathrm{~kg}$ p.c. (produto comercial); 0,8kg pc; 2,00L pc; 2,00L pc; 2,50L pc; 1,70L pc; 2,50L pc por $100 \mathrm{~kg}$ de sementes, respectivamente. Todos os inseticidas utilizados são sistêmicos. Cada parcela experimental foi constituída por um vaso com $6 \mathrm{~kg}$ de substrato e cada tratamento repetido cinco vezes, totalizando 40 plantas. Em cada vaso, foram semeadas três sementes de milho, variedade "Pop Zélia”, tratadas ou não com inseticidas. Após a emergência, realizouse o desbaste, deixando-se uma planta por vaso.

Aos dois dias após a emergência, as plântulas foram cobertas com gaiolas de confinamento e grupos de dez adultos da cigarrinha $\boldsymbol{D}$. maidis, sadios e com idade entre duas a três semanas, foram colocados para se alimentar nessas plântulas, conforme metodologia descrita por OLIVEIRA \& LOPES (2004). Após a introdução das cigarrinhas, nas gaiolas, foi concedido um período de aclimatação ao ambiente de confinamento de 15 minutos. Findo esse período, foram realizadas avaliações de mortalidade nos seguintes intervalos de tempo: 1, 2, 4, 8, 12 e 24h. Foram considerados mortos apenas aqueles insetos que não mais apresentavam movimentos de pernas e asas. Em intervalos de sete dias, novos grupos de cigarrinhas sadias foram colocados sobre as plantas, entre o segundo e o $58^{\circ}$ dia após a emergência, e os procedimentos de avaliação foram repetidos.

Utilizou-se o delineamento experimental inteiramente ao acaso com medidas repetidas no tempo. Os dados de mortalidade (\%) dos tratamentos e da testemunha foram utilizados para o cálculo da eficiência dos inseticidas por meio da fórmula de Abbott $\mathrm{Ma}=$ $(\mathrm{Mt}-\mathrm{Mc}) /(100-\mathrm{Mc}) \times 100$, em que Ma = mortalidade corrigida em função do tratamento testemunha; $\mathrm{Mt}=$ mortalidade observada no tratamento com o inseticida e $\mathrm{Mc}=$ mortalidade observada no tratamento testemunha (ABBOTT, 1925). Os dados foram então transformados segundo arcsen $\sqrt{x / n}$, sendo que, no caso de $x=0$ e $x=n$, utilizouse arcsen $\sqrt{1 / 4 n}$ earcsen $\sqrt{4 n-1 / 4 n}$ respectivamente (PIMENTEL-GOMES, 2000). Os dados foram submetidos à análise de variância e as médias comparadas pelo teste de Scott-Knott a 5\% de probabilidade de erro, pelo programa estatístico SISVAR 4.3(FERREIRA, 2000).

Com exceção de carbofuran $+\mathrm{Zn}$, todos os inseticidas mostraram alta eficiência no controle de $\boldsymbol{D}$. maidis, aos dois dias após a emergência, causando mortalidade dos espécimes entre 94 e 100\%. A eficiência desse controle foi reduzida em função das datas de avaliação (Tabela 1). O thiamethoxan e o imidacloprid foram os mais eficientes e mostraram resultados semelhantes em todas as datas de avaliação, exceto aos 51 dias. A mortalidade causada por esses dois inseticidas foi superior a $90 \%$ até o $16^{\circ}$ dia e igual ou superior a $70 \%$ até o 30 o dia de avaliação. No Brasil, thiamethoxan e imidacloprid, além de clotianidina, estão registrados no Ministério da Agricultura, Pecuária e Abastecimento (MAPA) para controle de $\boldsymbol{D}$. maidis.

Em campo, BHIRUD \& PITRE (1972) relataram a eficiência de carbofuran sobre $\boldsymbol{D}$. maidis, controlando $100 \%$ dos insetos até 35 dias após aplicação. Por outro lado, PERFECTO (1990) relatou ineficiência desse produto para o controle da cigarrinhado-milho. WAQUIL \& CRUZ (1994), utilizando 6 inseticidas em tratamento de sementes de milho, não observaram redução na infestação das plantas pela cigarrinha $\boldsymbol{D}$. maidis, em campo.

Aos dois dias após a emergência das plantas, os produtos thiamethoxan, imidacloprid e

Tabela 1 - Eficiência média de controle (calculada pela fórmula de Abbott) de adultos de Dalbulus maidis confinados em plantas provenientes de sementes de milho-pipoca cultivar “Pop Zélia” tratadas com inseticidas, em condições de viveiro telado.

\begin{tabular}{|c|c|c|c|c|c|c|c|c|c|c|c|c|c|c|c|c|c|c|}
\hline \multirow{2}{*}{$\begin{array}{l}\text { Inseticida } \\
\text { thiamethoxan }\end{array}$} & \multicolumn{2}{|c|}{2 dias $^{1}$} & \multicolumn{2}{|c|}{9 dias } & \multicolumn{2}{|c|}{16 dias } & \multicolumn{2}{|c|}{23 dias } & \multicolumn{2}{|c|}{30 dias } & \multicolumn{2}{|c|}{37 dias } & \multicolumn{2}{|c|}{44 dias } & \multicolumn{2}{|c|}{51 dias } & \multicolumn{2}{|c|}{58 dias } \\
\hline & 100 & $a A^{2}$ & 100 & $\mathrm{aA}$ & 98 & $\mathrm{aA}$ & 74 & $\mathrm{aB}$ & 76 & $\mathrm{aB}$ & 58 & $\mathrm{aC}$ & 36 & $\mathrm{aD}$ & 22 & $\mathrm{aE}$ & 6 & $\mathrm{aF}$ \\
\hline imidacloprid & 100 & $\mathrm{aA}$ & 100 & $\mathrm{aA}$ & 92 & $\mathrm{aA}$ & 80 & $\mathrm{aB}$ & 70 & $\mathrm{aB}$ & 68 & $\mathrm{aB}$ & 24 & $\mathrm{aC}$ & 8 & $\mathrm{bD}$ & 2 & $\mathrm{aD}$ \\
\hline thiodicarb $+\mathrm{zn}$ & 100 & $\mathrm{aA}$ & 98 & $\mathrm{aA}$ & 64 & $\mathrm{bB}$ & 16 & $\mathrm{bC}$ & 8 & $\mathrm{bD}$ & 0 & $\mathrm{bD}$ & 0 & $\mathrm{bD}$ & 0 & $\mathrm{bD}$ & 0 & $\mathrm{aD}$ \\
\hline thiodicarb & 100 & $\mathrm{aA}$ & 98 & $\mathrm{aA}$ & 56 & $\mathrm{bB}$ & 16 & $\mathrm{bC}$ & 4 & $\mathrm{bC}$ & 0 & $\mathrm{bD}$ & 0 & $\mathrm{bD}$ & 0 & $\mathrm{bD}$ & 0 & $\mathrm{aD}$ \\
\hline carbofuran & 100 & $\mathrm{aA}$ & 80 & bB & 0 & $\mathrm{cC}$ & 0 & $\mathrm{cC}$ & 0 & $\mathrm{bC}$ & 0 & $\mathrm{bC}$ & 0 & $\mathrm{bC}$ & 0 & bC & 0 & $\mathrm{aC}$ \\
\hline carbosulfan & 94 & $\mathrm{aA}$ & 14 & $\mathrm{cB}$ & 0 & $\mathrm{cC}$ & 0 & $\mathrm{cC}$ & 0 & bC & 0 & $\mathrm{bC}$ & 0 & bC & 0 & $\mathrm{bC}$ & 0 & $\mathrm{aC}$ \\
\hline carbofuran $+\mathrm{zn}$ & 8 & bA & 2 & $\mathrm{dA}$ & 0 & $\mathrm{cA}$ & 0 & $\mathrm{cA}$ & 0 & $\mathrm{bA}$ & 0 & bA & 0 & bA & 0 & bA & 0 & $\mathrm{aA}$ \\
\hline
\end{tabular}

\footnotetext{
${ }^{1}$ Dias após a emergência das plantas.

2 Médias seguidas de mesma letra minúscula nas colunas e maiúsculas nas linhas não diferem pelo teste de Scott-Knott a $5 \%$ de probabilidade de erro.
} 
carbofuran tiveram uma eficiência de controle de $100 \%$ dos insetos após um período de confinamento de quatro horas. Thiodicarb + Zn e thiodicarb mostraram 98 e $92 \%$ de eficiência no controle das cigarrinhas com oito horas de confinamento, e 100\% com 12 horas (Tabela 2). Aos 23 e 30 dias após a emergência das plantas, eficiência igual ou superior a $70 \%$, para thiamethoxan e imidacloprid, só foi alcançada após 24 horas de confinamento dos insetos. O inseticida carbosulfan apresentou boa eficiência de controle de D. maidis apenas aos dois dias após a emergência, com 12 horas de confinamento (Tabelas 1, 2 e 3).

Os inseticidas utilizados, neste experimento, são sistêmicos e atuam sobre as cigarrinhas quando

Tabela 2 - Eficiência média acumulada (calculada pela fórmula de Abbott) dos inseticidas utilizados em tratamento de sementes de milhopipoca cultivar "Pop Zélia" no controle de adultos de Dalbulus maidis, avaliada aos 2, 9, 16, 23 e 30 dias após a emergência das plantas e em diferentes intervalos de tempo, em condições de viveiro telado.

\begin{tabular}{|c|c|c|c|c|c|c|c|c|c|c|c|c|c|c|}
\hline \multirow[b]{2}{*}{$\begin{array}{l}\text { Dia e tempo de } \\
\text { avaliação }^{2}\end{array}$} & \multicolumn{14}{|c|}{ Média acumulada de eficiência de controle ${ }^{1}$} \\
\hline & \multicolumn{2}{|c|}{ thiamethoxan } & \multicolumn{2}{|c|}{ imidaclhorprid } & \multicolumn{2}{|c|}{ thiodicarb } & \multicolumn{2}{|c|}{ thiodicarb + zn } & \multicolumn{2}{|c|}{ carbofuran } & \multicolumn{2}{|c|}{ carbosulfan } & \multicolumn{2}{|c|}{ carbofuran $+\mathrm{zn}$} \\
\hline \multicolumn{15}{|l|}{ Dia 2} \\
\hline $1 \mathrm{~h}$ & 30 & $b^{3}$ & 80 & $\mathrm{a}$ & 8 & C & 6 & $\mathrm{~b}$ & 26 & $\mathrm{~b}$ & 2 & c & 0 & $\mathrm{a}$ \\
\hline $2 \mathrm{~h}$ & 54 & $\mathrm{~b}$ & 96 & $\mathrm{a}$ & 20 & C & 24 & $\mathrm{~b}$ & 42 & $\mathrm{~b}$ & 10 & $\mathrm{C}$ & 0 & $\mathrm{a}$ \\
\hline $4 \mathrm{~h}$ & 100 & $\mathrm{a}$ & 100 & $\mathrm{a}$ & 68 & $b$ & 76 & $\mathrm{a}$ & 100 & $\mathrm{a}$ & 28 & $\mathrm{~b}$ & 0 & $\mathrm{a}$ \\
\hline $8 \mathrm{~h}$ & 100 & $\mathrm{a}$ & 100 & $\mathrm{a}$ & 98 & $\mathrm{a}$ & 92 & $\mathrm{a}$ & 100 & $\mathrm{a}$ & 46 & $\mathrm{~b}$ & 2 & $\mathrm{a}$ \\
\hline $12 \mathrm{~h}$ & 100 & $\mathrm{a}$ & 100 & $\mathrm{a}$ & 100 & $\mathrm{a}$ & 100 & $\mathrm{a}$ & 100 & $\mathrm{a}$ & 78 & $\mathrm{a}$ & 2 & $\mathrm{a}$ \\
\hline $24 \mathrm{~h}$ & 100 & $\mathrm{a}$ & 100 & $\mathrm{a}$ & 100 & $\mathrm{a}$ & 100 & $\mathrm{a}$ & 100 & $\mathrm{a}$ & 94 & $\mathrm{a}$ & 8 & $\mathrm{a}$ \\
\hline \multicolumn{15}{|l|}{ Dia 9} \\
\hline $1 \mathrm{~h}$ & 6 & $\mathrm{C}$ & 26 & $\mathrm{C}$ & 0 & $\mathrm{C}$ & 4 & $\mathrm{C}$ & 2 & $\mathrm{C}$ & 0 & $\mathrm{a}$ & 0 & $\mathrm{a}$ \\
\hline $2 \mathrm{~h}$ & 22 & c & 50 & c & 10 & c & 24 & c & 8 & c & 0 & $\mathrm{a}$ & 0 & $\mathrm{a}$ \\
\hline $4 \mathrm{~h}$ & 50 & b & 74 & b & 52 & $\mathrm{~b}$ & 56 & $\mathrm{~b}$ & 44 & $\mathrm{~b}$ & 2 & $\mathrm{a}$ & 0 & $\mathrm{a}$ \\
\hline $8 \mathrm{~h}$ & 94 & $\mathrm{a}$ & 92 & $\mathrm{a}$ & 90 & $\mathrm{a}$ & 90 & $\mathrm{a}$ & 68 & $\mathrm{a}$ & 6 & $\mathrm{a}$ & 2 & $\mathrm{a}$ \\
\hline $12 \mathrm{~h}$ & 100 & $\mathrm{a}$ & 100 & $\mathrm{a}$ & 90 & $\mathrm{a}$ & 94 & $\mathrm{a}$ & 74 & $\mathrm{a}$ & 6 & $\mathrm{a}$ & 2 & $\mathrm{a}$ \\
\hline $24 \mathrm{~h}$ & 100 & $\mathrm{a}$ & 100 & $\mathrm{a}$ & 98 & $\mathrm{a}$ & 98 & $\mathrm{a}$ & 80 & $\mathrm{a}$ & 14 & $\mathrm{a}$ & 2 & $\mathrm{a}$ \\
\hline \multicolumn{15}{|l|}{ Dia 16} \\
\hline $1 \mathrm{~h}$ & 2 & $\mathrm{~b}$ & 20 & $\mathrm{~b}$ & 0 & $\mathrm{C}$ & 2 & $\mathrm{~b}$ & 0 & $\mathrm{a}$ & 0 & $\mathrm{a}$ & 0 & $\mathrm{a}$ \\
\hline $2 \mathrm{~h}$ & 6 & $\mathrm{~b}$ & 40 & b & 2 & c & 2 & $\mathrm{~b}$ & 0 & $\mathrm{a}$ & 0 & $\mathrm{a}$ & 0 & $\mathrm{a}$ \\
\hline $4 \mathrm{~h}$ & 14 & b & 46 & b & 4 & C & 4 & $\mathrm{~b}$ & 0 & $\mathrm{a}$ & 0 & $\mathrm{a}$ & 0 & $\mathrm{a}$ \\
\hline $8 \mathrm{~h}$ & 84 & $\mathrm{a}$ & 84 & $\mathrm{a}$ & 22 & $b$ & 14 & $\mathrm{~b}$ & 0 & $\mathrm{a}$ & 0 & $\mathrm{a}$ & 0 & $\mathrm{a}$ \\
\hline $12 \mathrm{~h}$ & 94 & $\mathrm{a}$ & 92 & $\mathrm{a}$ & 22 & $\mathrm{~b}$ & 14 & $\mathrm{~b}$ & 0 & $\mathrm{a}$ & 0 & $\mathrm{a}$ & 0 & $\mathrm{a}$ \\
\hline $24 \mathrm{~h}$ & 98 & $\mathrm{a}$ & 92 & $\mathrm{a}$ & 66 & $\mathrm{a}$ & 56 & $\mathrm{a}$ & 0 & $\mathrm{a}$ & 0 & $\mathrm{a}$ & 0 & $\mathrm{a}$ \\
\hline \multicolumn{15}{|l|}{ Dia 23} \\
\hline $1 \mathrm{~h}$ & 0 & $\mathrm{C}$ & 6 & $\mathrm{~b}$ & 0 & $\mathrm{a}$ & 0 & $\mathrm{~b}$ & 0 & & 0 & & 0 & \\
\hline $2 \mathrm{~h}$ & 0 & c & 14 & $\mathrm{~b}$ & 0 & $\mathrm{a}$ & 0 & $\mathrm{~b}$ & 0 & $\mathrm{a}$ & 0 & $\mathrm{a}$ & 0 & $\mathrm{a}$ \\
\hline $4 \mathrm{~h}$ & 2 & c & 18 & $\mathrm{~b}$ & 0 & $\mathrm{a}$ & 0 & $\mathrm{~b}$ & 0 & $\mathrm{a}$ & 0 & $\mathrm{a}$ & 0 & $\mathrm{a}$ \\
\hline $8 \mathrm{~h}$ & 18 & c & 18 & $\mathrm{~b}$ & 0 & $\mathrm{a}$ & 2 & $\mathrm{~b}$ & 0 & $\mathrm{a}$ & 0 & $\mathrm{a}$ & 0 & $\mathrm{a}$ \\
\hline $12 \mathrm{~h}$ & 44 & $\mathrm{~b}$ & 28 & $\mathrm{~b}$ & 2 & $\mathrm{a}$ & 2 & $\mathrm{~b}$ & 0 & $\mathrm{a}$ & 0 & $\mathrm{a}$ & 0 & $\mathrm{a}$ \\
\hline $24 h$ & 74 & $\mathrm{a}$ & 80 & $\mathrm{a}$ & 16 & $\mathrm{a}$ & 16 & $\mathrm{a}$ & 0 & $\mathrm{a}$ & 0 & $\mathrm{a}$ & 0 & $\mathrm{a}$ \\
\hline \multicolumn{15}{|l|}{ Dia 30} \\
\hline $1 \mathrm{~h}$ & 0 & $\mathrm{C}$ & 0 & $\mathrm{C}$ & 2 & $\mathrm{a}$ & 0 & $\mathrm{a}$ & 0 & $\mathrm{a}$ & 0 & $\mathrm{a}$ & 0 & $\mathrm{a}$ \\
\hline $2 \mathrm{~h}$ & 4 & C & 4 & c & 2 & $\mathrm{a}$ & 0 & $\mathrm{a}$ & 0 & $\mathrm{a}$ & 0 & $\mathrm{a}$ & 0 & $\mathrm{a}$ \\
\hline $4 \mathrm{~h}$ & 6 & c & 8 & c & 4 & $\mathrm{a}$ & 2 & $\mathrm{a}$ & 0 & $\mathrm{a}$ & 0 & $\mathrm{a}$ & 0 & $\mathrm{a}$ \\
\hline $8 \mathrm{~h}$ & 14 & c & 12 & c & 4 & $\mathrm{a}$ & 2 & $\mathrm{a}$ & 0 & $\mathrm{a}$ & 0 & $\mathrm{a}$ & 0 & $\mathrm{a}$ \\
\hline $12 \mathrm{~h}$ & 36 & $\mathrm{~b}$ & 34 & b & 4 & $\mathrm{a}$ & 4 & $\mathrm{a}$ & 0 & $\mathrm{a}$ & 0 & $\mathrm{a}$ & 0 & $\mathrm{a}$ \\
\hline $24 \mathrm{~h}$ & 76 & $\mathrm{a}$ & 70 & $\mathrm{a}$ & 8 & $\mathrm{a}$ & 4 & $\mathrm{a}$ & 0 & $\mathrm{a}$ & 0 & $\mathrm{a}$ & 0 & $\mathrm{a}$ \\
\hline
\end{tabular}

15 plantas/tratamento e 10 cigarrinhas/planta.

${ }^{2}$ Dias após a emergência das plantas e tempo de confinamento das cigarrinhas nas plantas.

${ }^{3}$ Médias seguidas de mesma letra nas colunas e dentro de cada data de avaliação não diferem pelo teste de Scott-Knott a 5\% de probabilidade de erro. 
Tabela 3 - Eficiência média acumulada (calculada pela fórmula de Abbott) dos inseticidas utilizados em tratamento de sementes de milhopipoca cultivar "Pop Zélia” no controle de adultos de Dalbulus maidis, avaliada aos 37, 44, 51 e 58 dias após a emergência das plantas e em diferentes intervalos de tempo, em condições de viveiro telado.

\begin{tabular}{|c|c|c|c|c|c|c|c|c|c|c|c|c|c|c|}
\hline \multirow[b]{2}{*}{$\begin{array}{l}\text { Dia e tempo de } \\
\text { avaliação }^{2}\end{array}$} & \multicolumn{14}{|c|}{ Média acumulada de eficiência de controle ${ }^{1}$} \\
\hline & \multicolumn{2}{|c|}{ thiamethoxan } & \multicolumn{2}{|c|}{ imidaclhorprid } & \multicolumn{2}{|c|}{ thiodicarb } & \multicolumn{2}{|c|}{ thiodicarb + zn } & \multicolumn{2}{|c|}{ carbofuran } & \multicolumn{2}{|c|}{ carbosulfan } & \multicolumn{2}{|c|}{ carbofuran $+\mathrm{zn}$} \\
\hline \multirow{2}{*}{\multicolumn{15}{|c|}{ Dia 37}} \\
\hline & & & & & & & & & & & & & & \\
\hline $1 \mathrm{~h}$ & 0 & $c^{3}$ & 2 & $\mathrm{~b}$ & 0 & a & 0 & $\mathrm{a}$ & 0 & $\mathrm{a}$ & 0 & $\mathrm{a}$ & 0 & $\mathrm{a}$ \\
\hline $2 \mathrm{~h}$ & 0 & c & 2 & $\mathrm{~b}$ & 0 & a & 0 & $\mathrm{a}$ & 0 & $\mathrm{a}$ & 0 & $\mathrm{a}$ & 0 & $\mathrm{a}$ \\
\hline $4 \mathrm{~h}$ & 4 & c & 8 & $\mathrm{~b}$ & 0 & a & 0 & $\mathrm{a}$ & 0 & $\mathrm{a}$ & 0 & $\mathrm{a}$ & 0 & $\mathrm{a}$ \\
\hline $8 \mathrm{~h}$ & 8 & c & 12 & b & 0 & $\mathrm{a}$ & 0 & $\mathrm{a}$ & 0 & $\mathrm{a}$ & 0 & $\mathrm{a}$ & 0 & $\mathrm{a}$ \\
\hline $12 \mathrm{~h}$ & 28 & b & 28 & b & 0 & $\mathrm{a}$ & 0 & $\mathrm{a}$ & 0 & $\mathrm{a}$ & 0 & $\mathrm{a}$ & 0 & $\mathrm{a}$ \\
\hline $24 \mathrm{~h}$ & 58 & $\mathrm{a}$ & 68 & $\mathrm{a}$ & 0 & $\mathrm{a}$ & 0 & $\mathrm{a}$ & 0 & $\mathrm{a}$ & 0 & $\mathrm{a}$ & 0 & $\mathrm{a}$ \\
\hline \multicolumn{15}{|l|}{ Dia 44} \\
\hline $1 \mathrm{~h}$ & 0 & b & 0 & $\mathrm{a}$ & 0 & $\mathrm{a}$ & 0 & $\mathrm{a}$ & 0 & $\mathrm{a}$ & 0 & $\mathrm{a}$ & 0 & $\mathrm{a}$ \\
\hline $2 \mathrm{~h}$ & 0 & b & 0 & $\mathrm{a}$ & 0 & a & 0 & $\mathrm{a}$ & 0 & $\mathrm{a}$ & 0 & $\mathrm{a}$ & 0 & $\mathrm{a}$ \\
\hline $4 \mathrm{~h}$ & 0 & b & 0 & $\mathrm{a}$ & 0 & $\mathrm{a}$ & 0 & $\mathrm{a}$ & 0 & $\mathrm{a}$ & 0 & $\mathrm{a}$ & 0 & $\mathrm{a}$ \\
\hline $8 \mathrm{~h}$ & 2 & b & 0 & $\mathrm{a}$ & 0 & $\mathrm{a}$ & 0 & $\mathrm{a}$ & 0 & $\mathrm{a}$ & 0 & $\mathrm{a}$ & 0 & $\mathrm{a}$ \\
\hline $12 \mathrm{~h}$ & 16 & $\mathrm{a}$ & 8 & $\mathrm{a}$ & 0 & $\mathrm{a}$ & 0 & $\mathrm{a}$ & 0 & $\mathrm{a}$ & 0 & $\mathrm{a}$ & 0 & $\mathrm{a}$ \\
\hline $24 \mathrm{~h}$ & 36 & $\mathrm{a}$ & 24 & $\mathrm{a}$ & 0 & $\mathrm{a}$ & 0 & $\mathrm{a}$ & 0 & $\mathrm{a}$ & 0 & $\mathrm{a}$ & 0 & $\mathrm{a}$ \\
\hline \multicolumn{15}{|l|}{ Dia 51} \\
\hline $1 \mathrm{~h}$ & 0 & $\mathrm{a}$ & 0 & $\mathrm{a}$ & 0 & $\mathrm{a}$ & 0 & $\mathrm{a}$ & 0 & $\mathrm{a}$ & 0 & $\mathrm{a}$ & 0 & $\mathrm{a}$ \\
\hline $2 \mathrm{~h}$ & 0 & $\mathrm{a}$ & 0 & $\mathrm{a}$ & 0 & $\mathrm{a}$ & 0 & $\mathrm{a}$ & 0 & $\mathrm{a}$ & 0 & $\mathrm{a}$ & 0 & $\mathrm{a}$ \\
\hline $4 \mathrm{~h}$ & 0 & $\mathrm{a}$ & 0 & $\mathrm{a}$ & 0 & $\mathrm{a}$ & 0 & $\mathrm{a}$ & 0 & $\mathrm{a}$ & 0 & $\mathrm{a}$ & 0 & $\mathrm{a}$ \\
\hline $8 \mathrm{~h}$ & 0 & $\mathrm{a}$ & 0 & $\mathrm{a}$ & 0 & $\mathrm{a}$ & 0 & $\mathrm{a}$ & 0 & $\mathrm{a}$ & 0 & $\mathrm{a}$ & 0 & $\mathrm{a}$ \\
\hline $12 \mathrm{~h}$ & 0 & $\mathrm{a}$ & 0 & $\mathrm{a}$ & 0 & $\mathrm{a}$ & 0 & $\mathrm{a}$ & 0 & $\mathrm{a}$ & 0 & $\mathrm{a}$ & 0 & $\mathrm{a}$ \\
\hline $24 \mathrm{~h}$ & 22 & $\mathrm{a}$ & 8 & $\mathrm{a}$ & 0 & $\mathrm{a}$ & 0 & $\mathrm{a}$ & 0 & $\mathrm{a}$ & 0 & $\mathrm{a}$ & 0 & $\mathrm{a}$ \\
\hline \multicolumn{15}{|l|}{ Dia 58} \\
\hline $1 \mathrm{~h}$ & 0 & $\mathrm{a}$ & 0 & $\mathrm{a}$ & 0 & $\mathrm{a}$ & 0 & $\mathrm{a}$ & 0 & $\mathrm{a}$ & 0 & $\mathrm{a}$ & 0 & $\mathrm{a}$ \\
\hline $2 \mathrm{~h}$ & 0 & $\mathrm{a}$ & 0 & $\mathrm{a}$ & 0 & $\mathrm{a}$ & 0 & $\mathrm{a}$ & 0 & $\mathrm{a}$ & 0 & $\mathrm{a}$ & 0 & $\mathrm{a}$ \\
\hline $4 \mathrm{~h}$ & 0 & $\mathrm{a}$ & 0 & $\mathrm{a}$ & 0 & $\mathrm{a}$ & 0 & $\mathrm{a}$ & 0 & $\mathrm{a}$ & 0 & $\mathrm{a}$ & 0 & $\mathrm{a}$ \\
\hline $8 \mathrm{~h}$ & 0 & $\mathrm{a}$ & 0 & $\mathrm{a}$ & 0 & $\mathrm{a}$ & 0 & $\mathrm{a}$ & 0 & $\mathrm{a}$ & 0 & $\mathrm{a}$ & 0 & $\mathrm{a}$ \\
\hline $12 \mathrm{~h}$ & 0 & $\mathrm{a}$ & 0 & $\mathrm{a}$ & 0 & $\mathrm{a}$ & 0 & $\mathrm{a}$ & 0 & $\mathrm{a}$ & 0 & $\mathrm{a}$ & 0 & $\mathrm{a}$ \\
\hline $24 \mathrm{~h}$ & 6 & $\mathrm{a}$ & 2 & $\mathrm{a}$ & 0 & $\mathrm{a}$ & 0 & $\mathrm{a}$ & 0 & $\mathrm{a}$ & 0 & $\mathrm{a}$ & 0 & $\mathrm{a}$ \\
\hline
\end{tabular}

${ }^{1} 5$ plantas/tratamento e 10 cigarrinhas/planta.

${ }^{2}$ Dias após a emergência das plantas e tempo de confinamento das cigarrinhas nas plantas.

${ }^{3}$ Médias seguidas de mesma letra nas colunas e dentro de cada data de avaliação não diferem pelo teste de Scott-Knott a $5 \%$ de probabilidade de erro.

essas se alimentam da seiva contaminada. Observa-se que o tempo necessário para a morte dessas cigarrinhas pode ser importante com relação à possibilidade de transmissão dos fitopatógenos, quando se pretende reduzir a incidência dos enfezamentos. Portanto, em campo, é possível que mesmo as cigarrinhas que morram pela ingestão dos produtos inseticidas possam transmitir os molicutes para as plantas.

Os resultados obtidos permitem concluir que imidacloprid e thiamethoxan, em tratamento de sementes de milho, apresentam eficiência de controle para adultos de $\boldsymbol{D}$. maidis igual ou superior a 70\% até o 30 dia após a emergência das plantas, necessitando entre 4 e 24 horas para causar esses índices de mortalidade.

\section{AGRADECIMENTOS}

Agradecemos ao Convênio Embrapa/Unicentro Isabella Hendrix, pela concessão de bolsa de iniciação científica ao autor Marcus Canuto.

\section{REFERÊNCIAS}

ABBOTT, W.S. A method of computing the effectiveness of on insecticide. Journal Economic Entomology, Lanham, v.18, n.2, p.265-267, 1925.

Ciência Rural, v.38, n.1, jan-fev, 2008. 
BHIRUD, K.M.; PITRE, H.N. Bioactivity of systemic inseticides in corn: relations to leafhopper vector control and corn stunt disease incidence. Journal of Economic Entomology, Lanham, v.65, n.4, p.1134-1140, 1972.

FERREIRA, D.F. Análises estatísticas por meio do Sisvar para Windows versão 4.0. In: REUNIÃO ANUAL DA REGIÃO BRASILEIRA DA SOCIEDADE INTERNACIONAL DE BIOMETRIA, 45., 2000, São Carlos. Anais... São Carlos: UFSCAR, 2000. p.255-258.

LEGRAND, A.I.; POWER, A.G. Inoculation and acquisition of maize bushy stunt mycoplasma by its leafhopper vector Dalbulus maidis. Annals of Applied Biology, Wellesbourne, v.125, n.1, p.115-122, 1994.

LOPES, J.R.S.; OLIVEIRA, C.M. Vetores de vírus e molicutes em milho. In: OLIVEIRA, E.; OLIVEIRA, C.M. Doenças em milho: molicutes, vírus, vetores e mancha por Phaeosphaeria. Brasília: Embrapa Informação Tecnológica, 2004. Cap.2. p.35-60.

OLIVEIRA, C.M. Variação genética entre populações de Dalbulus maidis (DeLong \& Wolcott, 1923) (Hemiptera: Cicadellidae) e mecanismos de sobrevivência na entressafra do milho. 2000. 167f. Tese (Doutorado em Entomologia) - Curso de Pós-graduação em Entomologia,
Escola Superior de Agricultura "Luiz de Queiroz", Universidade de São Paulo.

OLIVEIRA, C.M.; LOPES, J.R.S. Técnicas para criação da cigarrinha-do-milho e inoculação de molicutes e vírus em planta. In: OLIVEIRA, E.; OLIVEIRA, C.M. Doenças em milho: molicutes, vírus, vetores e mancha por Phaeosphaeria. Brasília: Embrapa Informação Tecnológica. 2004. Cap.4, p.89116.

OLIVEIRA, E. et al. Enfezamento pálido e enfezamento vermelho na cultura do milho no Brasil Central. Fitopatologia Brasileira, Brasília, v.23, n.1, p.45-47,1998.

PERFECTO, I. Indirect and direct effects in a tropical agroecosystem: the maize-pest-ant system in Nicaragua. Ecology, Washington, v.71, n.6, p.2125-2134, 1990.

PIMENTEL-GOMES, F. Curso de estatística experimental. São Paulo: Nobel. 2000. 477p.

WAQUIL, J.M.; CRUZ, I. Impacto do tratamento de sementes de milho na infestação por Dalbulus maidis e Spodoptera frugiperda. In: CONGRESSO NACIONAL DE MILHO E SORGO, 20., 1994, Goiânia. Resumos... Goiânia: ABMS/ EMGOPA/EMBRAPA, CNPMS/ UFG/EMATER-GO, 1994. p.153. 